\title{
Further results regarding the degree Kirchhoff index of graphs
}

Lihua Feng, Guihai Yu, and Weijun Liu 


\title{
FURTHER RESULTS REGARDING THE DEGREE KIRCHHOFF INDEX OF GRAPHS
}

\author{
LIHUA FENG, GUIHAI YU, AND WEIJUN LIU \\ Received 24 September, 2013
}

\begin{abstract}
Let $G$ be a connected graph with vertex set $V(G)$. The degree Kirchhoff index of $G$ is defined as $S^{\prime}(G)=\sum_{\{u, v\} \subseteq V(G)} d(u) d(v) R(u, v)$, where $d(u)$ is the degree of vertex $u$, and $R(u, v)$ denotes the resistance distance between vertices $u$ and $v$. In this paper we obtain some upper and lower bounds for the degree Kirchhoff index of graphs. We also obtain some bounds for the Nordhaus-Gaddum-type result for the degree Kirchhoff index.
\end{abstract}

2010 Mathematics Subject Classification: 05C12; 05C50

Keywords: normalized Laplacian eigenvalues, degree Kirchhoff index, graphs

\section{INTRODUCTION}

In this paper all graphs considered are assumed to be connected. Let $G=$ $(V(G), E(G))$ be a simple undirected graph with $n=|V(G)|$ vertices and $m=$ $|E(G)|$ edges. For a vertex $u \in V(G), d(u)$ denote the degree of $u . \Delta=\max \{d(u) \mid u \in$ $V(G)\}, \delta=\min \{d(u) \mid u \in V(G)\}$. The distance $d(v, u)=d(u, v \mid G)$ between the vertices $v$ and $u$ of the graph $G$ is defined as the length of a shortest path between $v$ and $u$. The diameter of $G$, denoted by $D$, is the maximum distance between all pairs of vertices.

The Wiener index is defined as the sum of distances between all unordered pairs of vertices

$$
W(G)=\sum_{\{u, v\} \subseteq V(G)} d(u, v) .
$$

This molecular structure descriptor is one of the most used topological indices, well correlated with many physical and chemical properties of a variety of classes of chemical compounds. For details, see the survey paper [9].

This paper is supported by NNSFC $(11101245,11271208,61202362,11301302)$ and NSFSD (BS2013SF009, BS2010SF017, ZR2011AQ005). 
In [16], a weighted version of the Wiener index is established, called the Gutman index [22,23], which is defined as

$$
S(G)=\sum_{\{u, v\} \subseteq V(G)} d(u) d(v) d(u, v) .
$$

For tree [16] it holds that $S(G)=4 W(G)-(2 n-1)(n-1)$.

The Gutman index of graphs attracted attention only quite recently. Several bounds on the Gutman index are established in [1]. In [6] an asymptotic upper bound for $S(G)$ was reported. In [6] relations between the edge-Wiener index and Gutman index were established. The maximal and minimal Gutman indices of unicyclic and bicyclic graphs are determined in $[4,10]$ and $[3,12]$, respectively.

In 1993 Klein and Randić [19] introduced a new distance function named resistance distance, based on the theory of electrical networks. They viewed $G$ as an electrical network $N$ by replacing each edge of $G$ with a unit resistor. The resistance distance between the vertices $u$ and $v$ of the graph $G$, denoted by $R(u, v)=R(u, v \mid G)$, is then defined to be the effective resistance between the nodes $u$ and $v$ in $N$. Similar to the long recognized shortest-path distance, the resistance distance is also intrinsic to the graph, not only with some nice purely mathematical properties, but also with a substantial potential for chemical applications [18, 19, 25, 26].

The Kirchhoff index (or resistance index) is defined in analogy to the Wiener index as:

$$
K f(G)=\sum_{\{u, v\} \subseteq V(G)} R(u, v) .
$$

The Kirchhoff index is also much studied in the literature. Extremal unicyclic graphs with respect to the Kirchhoff index were determined in [15,27,28]. Deng also studied the Kirchhoff index of fully loaded unicyclic graphs [15] and graphs with many cut edges [8]. Zhou [30] characterized the extremal graphs with given matching number, connectivity, and minimal Kirchhoff index. Wang et al. [24] determined the first three minimal Kirchhoff indices among cacti.

Recently, a new index named degree Kirchhoff index was put forward in [2]. It is defined as

$$
S^{\prime}(G)=\sum_{\{u, v\} \subseteq V(G)} d(u) d(v) R(u, v) .
$$

Apparently, we can see that the degree Kirchhoff index may be viewed as the resistance-distance analogue of the Gutman index.

Let $L=\left(L_{u v}\right)_{n \times n}$ be the Laplacian matrix of the (connected) graph $G$. Its entry is

$$
L_{u v}= \begin{cases}d(u), & \text { if } u=v \\ -1, & \text { if } u \sim v \\ 0, & \text { otherwise }\end{cases}
$$


Suppose the Laplacian eigenvalues of $L(G)$ are $\mu_{1} \geq \mu_{2} \geq \cdots \geq \mu_{n-1}>\mu_{n}=0$. For details on Laplacian eigenvalues see $[13,14,21]$. Then a long time known result for the Kirchhoff index is [17]:

$$
K f(G)=n \sum_{i=1}^{n-1} \frac{1}{\mu_{i}} .
$$

In [5] Fan Chung defined the normalized Laplacian matrix $\mathscr{L}(G)=\left(\mathscr{L}_{u v}\right)_{n \times n}$ of a graph $G$ as follows:

$$
\mathscr{L}_{u v}= \begin{cases}1, & \text { if } u=v ; \\ -\frac{1}{\sqrt{d(u) d(v)}}, & \text { if } u \sim v ; \\ 0, & \text { otherwise, }\end{cases}
$$

We call eigenvalues of $\mathscr{L}(G)$ the normalized Laplacian eiganvalues of $G$, and the eigenvalues of $\mathscr{L}(G)$ are ordered by $\lambda_{1} \geq \lambda_{2} \geq \cdots \geq \lambda_{n-1} \geq \lambda_{n}=0$. One can find that $\mathscr{L}=D^{-1 / 2} L D^{-1 / 2}$.

A remarkable analogy between the Kirchhoff and degree Kirchhoff indices is the formula [2]:

$$
S^{\prime}(G)=2 m \sum_{i=1}^{n-1} \frac{1}{\lambda_{i}} .
$$

For the following consideration it is important to recall that $R(u, v)=R(v, u)$, $R(u, u)=0$ and that [19] $d(u, v) \geq R(u, v)$ with equality if and only if there is a unique path linking the vertices $u$ and $v$. Therefore, for trees, $K f(G)=W(G)$ and $S^{\prime}(G)=S(G)$.

In [31] the authors obtained the following result.

Theorem 1. Let $G$ be a connected (molecular) bipartite graph with $n \geq 2$ vertices and $m$ edges. Then

$$
S^{\prime}(G) \geq(2 n-3) m
$$

with equality if and only if $G \cong K_{r, n-r}$ for $1 \leq r \leq\left\lfloor\frac{n}{2}\right\rfloor$.

In [11] the upper and lower bounds the degree Kirchhoff index of unicyclic graphs is established. In this paper, we further study the degree Kirchhoff index of graphs and obtain several upper and lower bounds on the degree Kirchhoff index. We also obtain some bounds for the Nordhaus-Gaddum-type result for the degree Kirchhoff index.

\section{LEMMAS AND RESULTS}

Lemma 1 ([5]). Let $G$ be a graph on $n$ vertices. Then for $n \geq 2$,

$$
\lambda_{1} \geq \frac{n}{n-1},
$$

the equality holds if and only if $G \cong K_{n}$. 
Lemma 2 ([29]). Let $G$ be a graph with the largest degree $\Delta$. Then

$$
\lambda_{1}(G) \geq 1+\frac{1}{\Delta},
$$

with equality if and only if $G$ is a complete graph.

Recall that the chromatic number $\chi(G)$ of a graph $G$ is the minimum number of colors such that $G$ can be colored in a way such that no two adjacent vertices have the same color.

Lemma 3 ([5]). Let $G$ be a graph on $n$ vertices with chromatic number $\chi$. Then

$$
\lambda_{1}(G) \geq \frac{\chi}{\chi-1} .
$$

Lemma 4 ([7]). Let $G$ be a connected graph of order $n>2$. Then $\lambda_{2}=\cdots=\lambda_{n-1}$ if and only if $G \cong K_{n}$ or $G \cong K_{p, q}$.

Theorem 2. Let $G$ be a connected graph on $n>2$ vertices and $m$ edges. Then

$$
\begin{aligned}
& S^{\prime}(G) \geq 2 m\left(n-2+\frac{1}{n}\right), \\
& S^{\prime}(G) \geq 2 m\left(\frac{\Delta}{\Delta+1}+\frac{(n-2)^{2}}{n-1-\frac{1}{\Delta}}\right), \\
& S^{\prime}(G) \geq 2 m\left(\frac{\chi}{\chi+1}+\frac{(n-2)^{2}}{n-1-\frac{1}{\chi}}\right) .
\end{aligned}
$$

Each of the above equalities holds if and only if $G \cong K_{n}$.

Proof. From the definition, one has

$$
\begin{aligned}
\frac{1}{2 m} S^{\prime} & =\sum_{i=1}^{n-1} \frac{1}{\lambda_{i}} \\
& =\frac{1}{\lambda_{1}}+\sum_{i=2}^{n-1} \frac{1}{\lambda_{i}} \\
& \geq \frac{1}{\lambda_{1}}+\frac{(n-2)^{2}}{\sum_{i=2}^{n-1} \lambda_{i}} \\
& =\frac{1}{\lambda_{1}}+\frac{(n-2)^{2}}{n-\lambda_{1}} .
\end{aligned}
$$

Now we consider the function

$$
f(x)=\frac{1}{x}+\frac{(n-2)^{2}}{n-x} .
$$


It is easy to check that $\frac{d f(x)}{d x} \geq 0$ for $x \geq \frac{n}{n-1}$, with equality if and only if $x=\frac{n}{n-1}$. Therefore it follows that $f(x)$ is not decreasing with respect to $x$. Since $x \geq \frac{n}{n-1}$ from Lemma 1, we have

$$
f(x) \geq f\left(\frac{n}{n-1}\right)=n-2+\frac{1}{n} .
$$

This implies the result.

If the equality holds, then all the inequalities in the proof must be equalities. It follows that $\lambda_{2}=\cdots=\lambda_{n-1}$, and from Lemmas 4 and 1 we get the result. The reverse is easy to check.

Note that from the above proof and Lemmas 2,3 we can get

$$
\begin{aligned}
& f(x) \geq f\left(\frac{\Delta+1}{\Delta}\right)=\frac{\Delta}{\Delta+1}+\frac{(n-2)^{2}}{n-1-\frac{1}{\Delta}}, \\
& f(x) \geq f\left(\frac{\chi}{\chi-1}\right)=\frac{\chi}{\chi+1}+\frac{(n-2)^{2}}{n-1-\frac{1}{\chi}} .
\end{aligned}
$$

Therefore we obtain the desired two bounds involving the maximum degree or the chromatic number, which is larger than $f\left(\frac{n}{n-1}\right)$.

Fan Chung [5] obtained the following formula for the number of spanning trees of a graph based on the eigenvalues of the normalized Laplacian:

$$
t(G)=\frac{\prod_{v} d(v)}{\sum_{v} d(v)} \prod_{i=1}^{n-1} \lambda_{i}
$$

Theorem 3. Let $G$ be a connected graph with $n \geq 3$ vertices, $t$ spanning trees and maximum vertex degree $\Delta$. Then

$$
S^{\prime}(G) \geq 2 m\left(\frac{\Delta}{\Delta+1}+(n-2)\left(\frac{(\Delta+1) \prod_{v} d(v)}{2 m t \Delta}\right)^{\frac{1}{n-2}}\right),
$$

with equality if and only if $G \cong K_{n}$.

Proof. From the definition, one has

$$
\begin{aligned}
\frac{1}{2 m} S^{\prime} & =\sum_{i=1}^{n-1} \frac{1}{\lambda_{i}} \\
& =\frac{1}{\lambda_{1}}+\sum_{i=2}^{n-1} \frac{1}{\lambda_{i}} \\
& \geq \frac{1}{\lambda_{1}}+\frac{n-2}{\left(\prod_{i=2}^{n-1} \lambda_{i}\right)^{\frac{1}{n-2}}}
\end{aligned}
$$




$$
\begin{aligned}
& =\frac{1}{\lambda_{1}}+(n-2)\left(\frac{\lambda_{1}}{\prod_{i=1}^{n-1} \lambda_{i}}\right)^{\frac{1}{n-2}} \\
& =\frac{1}{\lambda_{1}}+(n-2)\left(\frac{\lambda_{1} \prod_{v} d(v)}{2 m t}\right)^{\frac{1}{n-2}} .
\end{aligned}
$$

Let

Then we have

$$
g(x)=\frac{1}{x}+(n-2)\left(\frac{x \prod_{v} d(v)}{2 m t}\right)^{\frac{1}{n-2}} .
$$

$$
\frac{d g(x)}{d x}=-\frac{1}{x^{2}}+\left(\frac{x \prod_{v} d(v)}{2 m t}\right)^{\frac{1}{n-2}-1}\left(\frac{\prod_{v} d(v)}{2 m t}\right)=-\frac{1}{x^{2}}+\frac{1}{x}\left(\frac{x \prod_{v} d(v)}{2 m t}\right)^{\frac{1}{n-2}} .
$$

Therefore $g(x)$ is non-decreasing for

$$
x \geq\left(\frac{2 m t}{\prod_{v} d(v)}\right)^{\frac{1}{n-1}} .
$$

Note that

$$
\left(\frac{2 m t}{\prod_{v} \operatorname{deg}(v)}\right)^{\frac{1}{n-1}}=\left(\prod_{i=1}^{n-1} \lambda_{i}\right)^{\frac{1}{n-1}} \leq \frac{\sum_{i=1}^{n-1} \lambda_{i}}{n-1}=\frac{n}{n-1} \leq \frac{\Delta+1}{\Delta} \leq \lambda_{1},
$$

it follows that

$$
g(x) \geq g\left(\frac{\Delta+1}{\Delta}\right)=\frac{\Delta}{\Delta+1}+(n-2)\left(\frac{(\Delta+1) \prod_{v} d(v)}{2 m t \Delta}\right)^{\frac{1}{n-2}} .
$$

This implies the result. The equality case is easy to see from Lemma 4.

One may note from the above proof that

$$
\frac{1}{2 m} S^{\prime}=\sum_{i=1}^{n-1} \frac{1}{\lambda_{i}} \geq \frac{n-1}{\left(\prod_{i=1}^{n-1} \lambda_{i}\right)^{\frac{1}{n-1}}}=(n-1)\left(\frac{\prod_{v} d(v)}{2 m t}\right)^{\frac{1}{n-1}},
$$

with equality if and only if $\lambda_{1}=\lambda_{2}=\cdots=\lambda_{n-1}$, that is $G \cong K_{n}$.

The above expression implies that $S^{\prime}(G)$ is strictly decreasing with respect to $t(G)$.

Before listing an upper bound for $t(G)$, we first recall one definition. For $k \geq 1$, a graph $G$ is $k$-connected if either $G$ is a complete graph $K_{k+1}$, or $G$ has at least $k+2$ vertices and contains no $(k-1)$-vertex cut. The maximal value of $k$ for which a connected graph $G$ is $k$-connected is the connectivity of $G$, denoted by $\kappa(G)$. If $G$ is disconnected, we define $\kappa(G)=0$.

If $G$ is a graph of order $n$, then $\kappa(G) \leq n-1$, with equality holding if and only if $G \cong K_{n}$. 
Recently, the following result for the spanning trees of graphs was obtained in [20].

Let $G$ be a connected graph of order $n$ with connectivity $\kappa(G)=k$. Then

$$
t(G) \leq k n^{k-1}(n-1)^{n-k-2} .
$$

The equality holds if and only if $G \cong\left(K_{1} \cup K_{n-k-1}\right) \vee K_{k}$.

By using this bound and expression (2.1), we can get the following bound involving connectivity.

Theorem 4. Let $G$ be a connected graph with $n \geq 3$ vertices. If $G$ has connectivity $\kappa(G)=k$, then

$$
S^{\prime} \geq 2 m(n-1)\left(\frac{\prod_{v} d(v)}{2 m k n^{k-1}(n-1)^{n-k-2}}\right)^{\frac{1}{n-1}},
$$

with equality if and only if $G \cong K_{n}$.

At last, we consider the Nordhaus-Gaddum-type result for the degree Kirchhoff index.

Theorem 5. Let $G$ be a connected (molecular) graph on $n \geq 5$ vertices with a connected complement $\bar{G}$. Then

$$
S^{\prime}(G)+S^{\prime}(\bar{G}) \geq \frac{(n-1)^{3}}{2} .
$$

Proof. From the result in Theorem 2, we have

$$
\begin{aligned}
S^{\prime}(G)+S^{\prime}(\bar{G}) \geq & 2 m\left(n-2+\frac{1}{n}\right)+\left(\left(\begin{array}{l}
n \\
2
\end{array}\right)-2 m\right)\left(n-2+\frac{1}{n}\right) \\
& =\left(\begin{array}{l}
n \\
2
\end{array}\right)\left(n-2+\frac{1}{n}\right)=\frac{(n-1)^{3}}{2} .
\end{aligned}
$$

This implies the result.

Lemma 5 ([2]). Let $G$ be a graph of order $n$, with $m$ edges and diameter $D$. For all $v_{i}, v_{j} \in V(G)(i \neq j)$, then

$$
R\left(v_{i}, v_{j}\right) \leq 2 m D\left(\frac{1}{d\left(v_{i}\right)}+\frac{1}{d\left(v_{j}\right)}\right) .
$$

Theorem 6. Let $G$ be a connected (molecular) graph on $n \geq 5$ vertices with diameter $D$. Then

$$
S^{\prime}(G) \leq 4 D(n-1) m^{2}
$$


Proof. From the definition, we have

$$
\begin{aligned}
S^{\prime}(G) & =\sum_{\{u, v\} \subseteq V(G)} d(u) d(v) R(u, v) \\
& \leq \sum_{\{u, v\} \subseteq V(G)} d(u) d(v) 2 m D\left(\frac{1}{d(u)}+\frac{1}{d(v)}\right) \\
& =2 m D \sum_{\{u, v\} \subseteq V(G)}(d(u)+d(v)) \\
& =2 m D(n-1) \sum_{u \in V(G)} d(u) \\
& =4 D(n-1) m^{2} .
\end{aligned}
$$

This implies the result.

Theorem 7. Let $G$ be a graph of order $n$ with maximum degree $\Delta$, and maximal Gutman index $S(G)$. Then the following holds:

$$
S^{\prime}(G) \leq\left(\begin{array}{c}
n+1 \\
3
\end{array}\right) \Delta^{3} .
$$

Proof. Since $d(u, v) \geq R(u, v)$ with equality if and only if there is a unique path linking the vertices $u$ and $v$ [19], we immediately get

$$
S^{\prime}(G)=\sum_{\{u, v\} \subseteq V(G)} d(u) d(v) R(u, v) \leq \sum_{\{u, v\} \subseteq V(G)} d(u) d(v) d(u, v)=S(G) .
$$

In [1] it is obtained that under the hypothesis of the theorem,

$$
S(G) \leq\left(\begin{array}{c}
n+1 \\
3
\end{array}\right) \Delta^{3} .
$$

The desired result is obtained.

We now present one example to illustrate that the above two bounds are incomparable. For the star graph $H=K_{1, n-1}$, bound (2.2) reads $8(n-1)^{3}$, while bound (4) is $(n-1)^{3}\left(\begin{array}{c}n+1 \\ 3\end{array}\right)$, showing that (2.2) is better than (2.3). For the path $P_{n}$, bound (3) reads $4(n-1)^{4}$, while bound $(2.3)$ is $8\left(\begin{array}{c}n+1 \\ 3\end{array}\right)$, showing that (2.3) is better than (2.2). Therefore the above two bounds are incomparable.

In light of Theorems 6, 7, we can establish the following two upper bounds for the Nordhaus-Gaddum-type results. 
Theorem 8. Let $G$ be a connected (molecular) graph on $n \geq 5$ vertices with a connected complement $\bar{G}$. Then

$$
S^{\prime}(G)+S^{\prime}(\bar{G}) \leq 4(n-1)^{2}\left(m^{2}+\left(\left(\begin{array}{l}
n \\
2
\end{array}\right)-m\right)^{2}\right) .
$$

Proof. Note that the diameter of any graph is at most $n-1$. From the result in Theorem 6, we have

$$
S^{\prime}(G)+S^{\prime}(\bar{G}) \leq 4(n-1)^{2}\left(m^{2}+\left(\left(\begin{array}{l}
n \\
2
\end{array}\right)-m\right)^{2}\right) .
$$

This implies the result.

Theorem 9. Let $G$ be a connected (molecular) graph on $n \geq 5$ vertices with a connected complement $\bar{G}$. Then

$$
S^{\prime}(G)+S^{\prime}(\bar{G}) \leq\left(\begin{array}{c}
n+1 \\
3
\end{array}\right)\left(\Delta^{3}+(n-1-\delta)^{3}\right) .
$$

Proof. From the result in Theorem 7, we have the result.

\section{FURTHER DISCUSSION}

As stated in Section 1, formulae (1.1) and (1.2) reveal that those two resistance distance based molecular descriptors have a close connection with the eigenvalues of some matrices associated with graphs. For a edge weighted connected graph $G$ with weight $c_{u v}$ on the edge $u v$, and $c(u)=\sum_{u w \in E(G)} c_{u w}$, we define $\bar{S}=$ $\sum_{\{u, v\} \subseteq V(G)} c(u) c(v) R(u, v)$. Then we may naturally ask that whether there exists a real symmetric and positive semidefinite matrix $P$ with eigenvalues

$$
\gamma_{1} \geq \gamma_{2} \geq \ldots \geq \gamma_{n-1}>\gamma_{n}=0
$$

such that $\bar{S}=\sum_{i=1}^{n-1} \frac{1}{\gamma_{i}}$ ?

Conversely, in general, let $M$ be a real symmetric and positive semidefinite matrix. Suppose its eigenvalues are

$$
\rho_{1} \geq \rho_{2} \geq \ldots \geq \rho_{n-1}>\rho_{n}=0,
$$

with their corresponding mutually orthogonal unit eigenvector

$$
\xi_{1}, \xi_{2}, \ldots \xi_{n-1}, \xi_{n}
$$

Now, we construct the matrix

$$
B=M+\frac{1}{a} \xi_{n}\left(\xi_{n}\right)^{t},
$$


where $a=\left(\xi_{n}\right)^{t} \xi_{n}$. We claim that the eigenvalues of $B$ are

$$
\rho_{1}, \rho_{2}, \ldots, \rho_{n-1}, 1 \text {. }
$$

In fact, we can check that, for $1 \leq i \leq n-1$,

$$
\begin{aligned}
B \xi_{i} & =\left(M+\frac{1}{a} \xi_{n}\left(\xi_{n}\right)^{t}\right) \xi_{i} \\
& =M \xi_{i}+\frac{1}{a} \xi_{n}\left(\xi_{n}\right)^{t} \xi_{i} \\
& =M \xi_{i} \\
& =\rho_{i} \xi_{i} .
\end{aligned}
$$

Similarly,

$$
B \xi_{n}=M \xi_{n}+\frac{1}{a} \xi_{n}\left(\xi_{n}\right)^{t} \xi_{n}=\frac{1}{a} \xi_{n}\left[\left(\xi_{n}\right)^{t} \xi_{n}\right]=\xi_{n}
$$

Since all the eigenvalues of $B$ are not zero, $B$ is non-singular. Therefore, its inverse $B^{-1}$ exists and its eigenvalues are $\frac{1}{\rho_{1}}, \frac{1}{\rho_{2}}, \ldots, \frac{1}{\rho_{n-1}}, 1$.

If we take $M=L=D-A$, and $\xi_{n}=1$, the all one vector, then we get Theorem 1 and Theorem 2 in [26]. And the formulae (1) is established.

If we take $M=\mathscr{L}=D^{-1 / 2} L D^{-1 / 2}$, and $\xi_{n}=D^{\frac{1}{2}} \mathbf{1}$, then we get some part of Theorem 3.1 in [2]. And the formulae (2) is established.

Another natural question is: for general $M$ described above, is there a connection between $\sum_{i=1}^{n-1} \frac{1}{\rho_{i}}$ and some (resistance) distance based molecular descriptor of graphs?

We leave it for further research.

\section{REFERENCES}

[1] V. Andova, D. Dimitrov, J. Fink, and R. Škrekovski, "Bounds on Gutman index," MATCH Commun. Math. Comput. Chem., vol. 67, no. 2, pp. 515-524, 2012.

[2] H. Chen and F. Zhang, "Resistance distance and the normalized Laplacian spectrum," Discrete Appl. Math., vol. 155, no. 5, pp. 654-661, 2007.

[3] S. Chen and W. Liu, "Extremal modified Schultz index of bicyclic graphs," MATCH Commun. Math. Comput. Chem., vol. 64, no. 3, pp. 767-782, 2010.

[4] S. Chen and W. Liu, "Extremal university graphs with respect to modified Schultz index," Util. Math., vol. 86, pp. 87-94, 2011.

[5] F. R. K. Chung, Spectral graph theory, ser. Regional Conference Series in Mathematics. Providence, RI: AMS, American Mathematical Society, 1997, vol. 92.

[6] P. Dankelmann, I. Gutman, S. Mukwembi, and H. C. Swart, "The edge-Wiener index of a graph," Discrete Math., vol. 309, no. 10, pp. 3452-3457, 2009.

[7] K. C. Das, A. Güngör, and Ş. Bozkurt, "On the normalized Laplacian eigenvalues of graphs," Ars Combin., in press.

[8] H. Deng, "On the minimum Kirchhoff index of graphs with given number of cut-edges," MATCH Commun. Math. Comput. Chem., vol. 63, no. 1, pp. 171-180, 2010.

[9] A. A. Dobrynin, R. Entringer, and I. Gutman, "Wiener index of trees: Theory and applications," Acta Appl. Math., vol. 66, no. 3, pp. 211-249, 2001. 
[10] L. Feng, "The Gutman index of unicyclic graphs," Discrete Math. Algorithms Appl., vol. 4, no. 3, pp. $1250031,8,2012$.

[11] L. Feng, I. Gutman, and G. Yu, "Degree Kirchhoff index of unicyclic graphs," MATCH Commun. Math. Comput. Chem., vol. 69, no. 3, pp. 629-648, 2013.

[12] L. Feng and W. Liu, "The maximal Gutman index of bicyclic graphs," MATCH Commun. Math. Comput. Chem., vol. 66, no. 2, pp. 699-708, 2011.

[13] R. Grone and R. Merris, "The Laplacian spectrum of a graph. II," SIAM J. Discrete Math., vol. 7, no. 2, pp. 221-229, 1994.

[14] R. Grone, R. Merris, and V. S. Sunder, "The Laplacian spectrum of a graph," SIAM J. Matrix Anal. Appl., vol. 11, no. 2, pp. 218-238, 1990.

[15] Q. Guo, H. Deng, and D. Chen, "The extremal Kirchhoff index of a class of unicyclic graphs," MATCH Commun. Math. Comput. Chem., vol. 61, no. 3, pp. 713-722, 2009.

[16] I. Gutman, "Selected properties of the Schultz molecular topological index," J. Chem. Inf. Comput. Sci., vol. 34, pp. 1087-1089, 1994.

[17] I. Gutman and B. Mohar, "The quasi-Wiener and the Kirchhoff indices coincide," J. Chem. Inf. Comput. Sci., vol. 36, pp. 982-985, 1996.

[18] D. J. Klein, “Graph geometry, graph metrics, and Wiener," Match, vol. 35, pp. 7-27, 1997.

[19] D. J. Klein and M. Randić, "Resistance distance," J. Math. Chem., vol. 12, pp. 81-95, 1993.

[20] J. Li, W. C. Shiu, and A. Chang, "The number of spanning trees of a graph," Appl. Math. Lett., vol. 23, no. 3, pp. 286-290, 2010.

[21] B. Mohar, "The Laplacian spectrum of graphs," in Graph theory, combinatorics, and applications, ser. Proceedings of the sixth quadrennial international conference on the theory and applications of graphs held at Western Michigan University, Kalamazoo, MI, USA, May 30-June 3, 1988 . New York: John Wiley \& Sons, Inc., 1991, vol. 2, pp. 871-898.

[22] R. Todeschini and V. Consonni, Handbook of Molecular Descriptors. Weinheim: Wiley-VCH, 2000.

[23] R. Todeschini and V. Consonni, Molecular Descriptors for Chemoinformatics. Weinheim: Wiley-VCH, 2009.

[24] H. Wang, H. Hua, and D. Wang, "Cacti with minimum, second-minimum, and third-minimum Kirchhoff indices," Math. Commun., vol. 15, no. 2, pp. 347-358, 2010.

[25] W. Xiao and I. Gutman, "On resistance matrices," MATCH Commun. Math. Comput. Chem., vol. 49, pp. 67-81, 2003.

[26] W. Xiao and I. Gutman, "Resistance distance and Laplacian spectrum," Theor. Chem. Acc., vol. 110, pp. 284-289, 2003.

[27] Y. Yang and X. Jiang, "Unicyclic graphs with extremal Kirchhoff index," MATCH Commun. Math. Comput. Chem., vol. 60, no. 1, pp. 107-120, 2008.

[28] W. Zhang and H. Deng, "The second maximal and minimal Kirchhoff indices of unicyclic graphs," MATCH Commun. Math. Comput. Chem., vol. 61, no. 3, pp. 683-695, 2009.

[29] X. Zhang, "On the Laplacian spectra of graphs," Ars Comb., vol. 72, pp. 191-198, 2004.

[30] B. Zhou and B. N. Trinajstić, "The Kirchhoff index and the matching number," Int. J. Quantum Chem., vol. 109, pp. 2978-2981, 2009.

[31] B. Zhou and N. Trinajstić, "On resistance-distance and Kirchhoff index," J. Math. Chem., vol. 46, no. 1, pp. 283-289, 2009.

Authors' addresses

Lihua Feng

Central South University, Department of Mathematics, Changsha, Hunan, 410083, P.R. China

E-mail address: fenglh@163.com 
Guihai Yu

Shandong Institute of Business \& Technology, School of Mathematics, Yantai, Shandong, P.R. China E-mail address: yuguihai@126.com

Weijun Liu

Central South University, Department of Mathematics, Changsha, Hunan, 410083, P.R. China

E-mail address: wjliu6210@163.com 